\title{
ALTERAÇÕES HIDROELETROLÍTICAS E ACIDOBÁSICAS NO PÓS-OPERATÓRIO IMEDIATO DO TRANSPLANTE DE FÍGADO: UMA REVISÃO QUALITATIVA
}

\author{
Hydroelectrolytic and acid-basic alterations on the immediate postoperative \\ liver transplantation: a qualitative review
}

\author{
Beatriz Costa Nava Martins ${ }^{1}$, Olival Cirilo Lucena da Fonseca Neto²
}

\section{RESUMO}

Introdução: O pós-operatório imediato do transplante de fígado (TF) é um período instável e que necessita de controle e monitorização do paciente. Alterações hidroeletrolíticas e acidobásicas são apenas uma das várias complicações do pós-operatório imediato de TF, mas chamam muita atenção, e precisam de rápido e correto manejo. Objetivo: Busca-se através desta revisão avaliar as principais alterações acidobásicas e hidroeletrolíticas no pós-operatório imediato dos pacientes transplantados de fígado. Métodos: Trata-se de uma revisão, um estudo qualitativo com pesquisa em bancos de dados do MEDLINE, através do National Center of Biotechnology Information, acessando o site Pubmed. Os critérios de inclusão foram: (1) publicações em língua inglesa, (2) serem artigos originais ou revisão de literatura e (3) estarem em concordância com tema. Resultados: Cerca de mais de $70 \%$ dos pacientes, segundo os artigos analisados, apresentam acidose metabólica no pós-operatório imediato do TF, associada à hipercloremia. Os níveis de sódio são os mais alterados com a hiponatremia. Em média, 20\% dos pacientes apresentam hipercalemia após transplante de fígado. Hipocalcemia, hipomagnesemia e hipofosfatemia são distúrbios muito frequentes nesses pacientes. Conclusões: Acidose metabólica associada à hipercloremia, hiponatremia, hipocalcemia e hipercalemia são os distúrbios mais comuns no pós-operatório mediato de TF.

Descritores: Desequilíbrio Ácido-base; Desequilíbrio Hidroeletrolítico; Período Pós-Operatório; Transplante de Fígado.

Instituições:

1 Faculdade de Ciências Médica, Hospital Oswaldo do Cruz, Recife/PE, Brasil

2 Unidade de Transplante de Fígado (UTF), Hospital Oswaldo Cruz, Recife/PE, Brasil

https://doi.org/10.53855/bjt.v24i4.432

Correspondência:

Beatriz Costa Nava Martins

E-mail: beatrizcnavam@gmail.com

\section{INTRODUÇÃO}

O período pós-operatório imediato é marcado por instabilidades e necessidade constante de monitorização, especialmente em cirurgias de grande porte. Uma das complicações frequentes nesse período são os distúrbios hidroeletrolíticos, devido à prolongada hidratação endovenosa e resposta corporal orgânica ao trauma. ${ }^{1}$ Alterações acidobásicas também apresentam destaque, sendo a acidose respiratória, metabólica ou mista o distúrbio mais comum nesses pacientes após o ato cirúrgico. ${ }^{2}$ 
No pós-operatório imediato das cirurgias de transplante de fígado (TF), os distúrbios hidroeletrolíticos são também comuns, sendo as alterações dos níveis de sódio as mais frequentes nesses pacientes; mas o monitoramento dos níveis de cálcio, potássio, cloro e magnésio também são muito importantes. ${ }^{3}$ Dentre os distúrbios acidobásicos, a acidose metabólica está presente em mais de $70 \%$ dos transplantados de fígado, e a influência dos altos níveis de cloro contribui muito para essa acidemia. ${ }^{4}$

As alterações hidroeletrolíticas e acidobásicas são apenas uma das variadas complicações do pósoperatório imediato de TF, mas chamam muita atenção, e precisam de rápido e correto manejo. Assim, esta revisão incluiu os principais estudos da literatura sobre os distúrbios hidroeletrolíticos e acidobásicos no pósoperatório imediato do transplante de fígado.

\section{MÉTODO}

Esta revisão é um estudo qualitativo sobre os distúrbios hidroeletrolíticos e acidobásicos no pós-operatório imediato do transplante de fígado, que reuniu artigos publicados entre 2005 e 2021.

Inicialmente, foram identificados registros sobre o tema: alterações hidroeletrolíticas e acidobásicas no pós-operatório imediato do transplante de fígado, com pesquisa em bancos de dados do MEDLINE através do National Center of Biotechnology Information, acessando o site https://pubmed.ncbi.nlm.nih.gov/. Em seguida, foram determinados os critérios de elegibilidade para realização da triagem dos estudos: os artigos com (1) textos completos disponíveis para análise foram analisados de acordo com os critérios de inclusão e exclusão. Os critérios de inclusão eram: (1) ser publicados em língua inglesa, (2) serem artigos originais ou revisão de literatura, e (3) estarem em concordância com tema. Os critérios de exclusão eram o não atendimento dos critérios de inclusão.

Dessa maneira, cerca de 40 artigos foram analisados e criteriosamente interpretados para categorização e melhor avaliação global destes, e assim, selecionar os 18 artigos finais. A forma de seleção dos artigos está disposta na figura 1 - fluxograma, a seguir.

Figura 1 - Fluxograma - Forma de seleção dos artigos

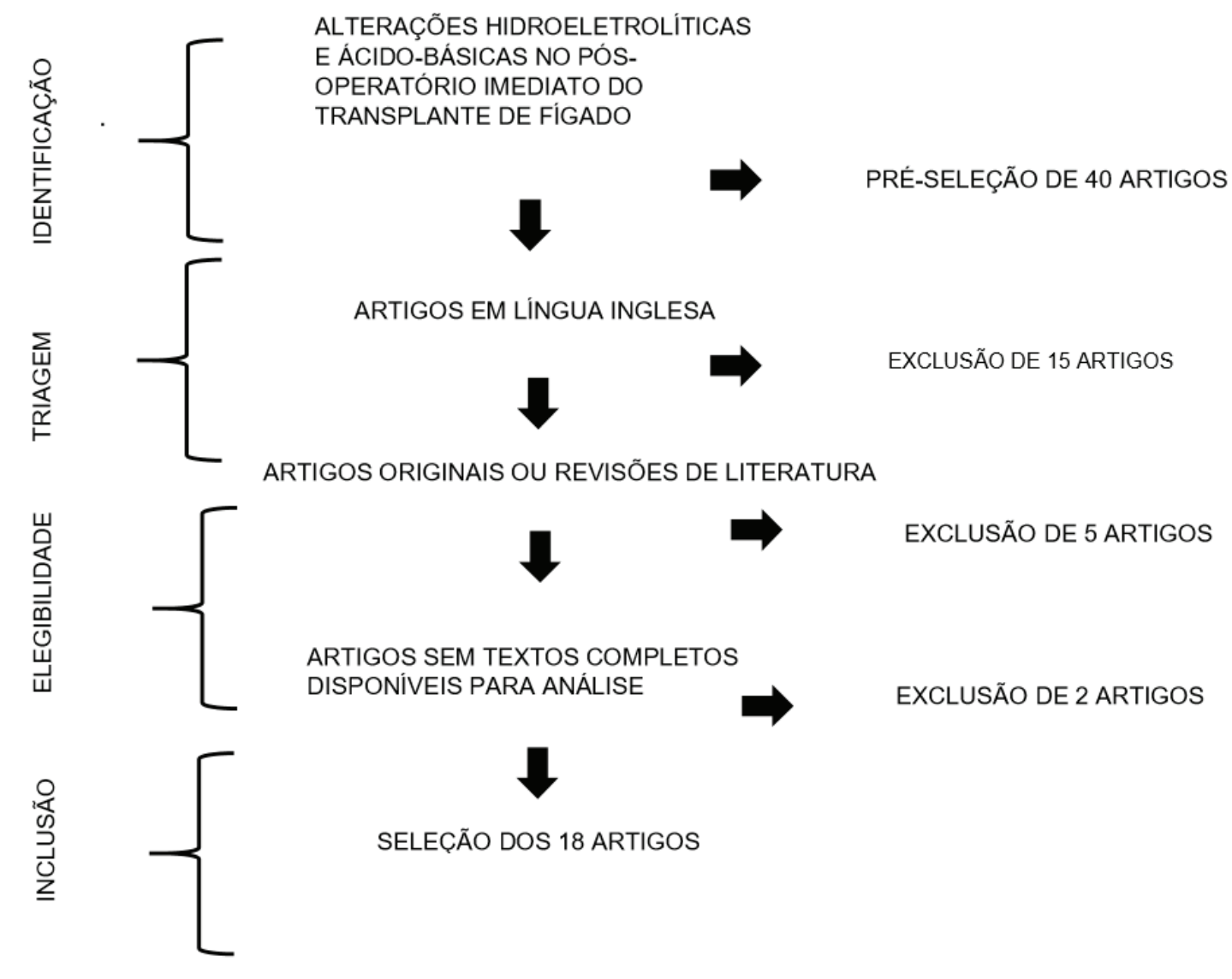




\section{RESULTADOS}

A maioria dos artigos selecionados trouxe resultados que seguiram a mesma linha de raciocínio: os pacientes, após o transplante de fígado, apresentam grandes instabilidades, com diversos tipos de complicações. Foram encontradas em $33,33 \%(6 / 18)$ dos artigos alterações hidroeletrolíticas e acidobásicas como uma das complicações mais frequentes, não apenas em pacientes transplantados, mas nos pacientes cirúrgicos em geral. ${ }^{1,2,5-8}$. Os 18 artigos selecionados foram detalhados na tabela 1 .

Tabela 1. Artigos incluidos na revisão e seus principais resultados.

\begin{tabular}{|c|c|c|}
\hline Artigos & Autor / Ano & Principais resultados \\
\hline $\begin{array}{l}\text { Hidratação e equilíbrio ácido-base } \\
\text { em pacientes cirúrgicos }\end{array}$ & $\begin{array}{l}\text { MORIYA, Takachi et al. } \\
\text { / } \\
2000\end{array}$ & $\begin{array}{l}\text { - Diante do manejo hídrico dos pacientes analisados, percebeu- } \\
\text { se que a alcalose respiratória no pré-operatório, e a acidose } \\
\text { respiratória no pós-operatório imediato são os distúrbios mais } \\
\text { relevantes nesses pacientes cirúrgicos. }\end{array}$ \\
\hline $\begin{array}{l}\text { Intensive care complications in liver } \\
\text { and multivisceral transplantation }\end{array}$ & $\begin{array}{l}\text { FAENZA, S. et al. / } \\
2005\end{array}$ & $\begin{array}{l}\text { - As complicações de pós-operatório do TF e multivisceral mais } \\
\text { comuns são: fracasso do enxerto, alterações hidroeletrolíticas, } \\
\text { complicações neurológicas, renais, respiratórias e infecciosas. }\end{array}$ \\
\hline $\begin{array}{l}\text { Equilíbrio hidroeletrolítico e } \\
\text { hidratação no paciente cirúrgico }\end{array}$ & $\begin{array}{l}\text { CENEVIVA, Reginaldo et al. } \\
\text { / } 2008\end{array}$ & $\begin{array}{l}\text { - O equilíbrio hidroeletrolítico é de grande importância ao } \\
\text { paciente cirúrgico, sendo definidor no pré, intra e pós-operatório. } \\
\text { É fundamental entender toda dinâmica hidroeletrolítica do } \\
\text { paciente para realizar a hidratação correta e necessária. }\end{array}$ \\
\hline $\begin{array}{l}\text { Fencl-Stewart analysis of acid-base } \\
\text { changes immediately after liver } \\
\text { transplantation. }\end{array}$ & $\begin{array}{l}\text { STORY, David A. et al. / } \\
2008\end{array}$ & $\begin{array}{l}\text { - O grupo transplantado de fígado em análise desenvolveu } \\
\text { acidose metabólica por influência de aumento de concentração } \\
\text { de cloreto e outros íons. Outros ânions são importantes no } \\
\text { quadro ácido-base. }\end{array}$ \\
\hline $\begin{array}{l}\text { Physical chemical approach versus } \\
\text { traditional technique in analyzing } \\
\text { blood gases and electrolytes during } \\
\text { liver transplant surgery. }\end{array}$ & ALI, Y. et al. / 2010 & $\begin{array}{l}\text { - Existe relação estreita entre nível de SIG e valores de pH. } \\
\text { - Menos fluidos com cloreto de sódio no peri-operatório, } \\
\text { diminuem a incidência de acidose hiperclorêmica no pós- } \\
\text { operatório. }\end{array}$ \\
\hline $\begin{array}{l}\text { Avaliação diagnóstica e prognóstica } \\
\text { dos distúrbios ácido-básicos em } \\
\text { pacientes críticos pelo método de } \\
\text { Stewart. }\end{array}$ & $\begin{array}{l}\text { BONIATTI, Márcio Manozzo. } \\
\text { / } 2010\end{array}$ & $\begin{array}{l}\text { - O método Stewart apresenta melhor precisão e melhora de } \\
\text { diagnóstico de alterações acidobásicas em pacientes críticos, em } \\
\text { comparação ao modelo tradicional. }\end{array}$ \\
\hline $\begin{array}{l}\text { The use of perioperative serial } \\
\text { blood lactate levels, the APACHE } \\
\text { II and the postoperative MELD as } \\
\text { predictors of early mortality after } \\
\text { liver transplantation }\end{array}$ & $\begin{array}{l}\text { BASILE-FILHO, Anibal et al., } \\
\text { / } 2011\end{array}$ & $\begin{array}{l}\text { - A mortalidade dos pacientes submetidos ao TF em até } 1 \text { mês } \\
\text { foi de } 17,3 \% \text {. } \\
\text { - O indicador MELD- pós TF dentre os avaliados, foi o melhor na } \\
\text { previsão sobre mortalidade. }\end{array}$ \\
\hline $\begin{array}{l}\text { Intensive care management of liver } \\
\text { transplanted patients. }\end{array}$ & $\begin{array}{l}\text { FELTRACCO, Paolo et al. / } \\
2011\end{array}$ & $\begin{array}{l}\text { - O pós-operatório do TF é multifatorial, por isso compreender } \\
\text { a terapia mais eficaz, conhecer os eventos de risco e as } \\
\text { complicações, diminui o tempo na UTI e melhora resultado geral } \\
\text { do transplante. }\end{array}$ \\
\hline $\begin{array}{l}\text { Physicochemical Evaluation } \\
\text { of Acid-Base Disorders After } \\
\text { Liver Transplantation and the } \\
\text { Contribution From Administered } \\
\text { Fluids }\end{array}$ & $\begin{array}{l}\text { BONIATTI, M. M. et al. / } \\
2013\end{array}$ & $\begin{array}{l}\text { - Em estudo } 72 \% \text { dos pacientes desenvolveram acidose } \\
\text { metabólica. A acidose foi atenuada pela queda de albumina. } \\
\text { - Hipercloremia é o fator principal contribuidor da acidose } \\
\text { metabólica nesses pacientes. }\end{array}$ \\
\hline $\begin{array}{l}\text { The interrelation between arterial } \\
\text { lactate levels and postoperative } \\
\text { outcome following liver } \\
\text { transplantation }\end{array}$ & JIPA, Lavinia N. et al., / 2014 & $\begin{array}{l}\text { - Lactato elevado está relacionado com maior perda sanguínea } \\
\text { na cirurgia, mais complicações como IRA e maior mortalidade. }\end{array}$ \\
\hline
\end{tabular}


Tabela 1. Artigos incluidos na revisão e seus principais resultados. (continuação)

\begin{tabular}{|c|c|c|}
\hline Artigos & Autor / Ano & Principais resultados \\
\hline $\begin{array}{l}\text { Chloride-liberal fluids are } \\
\text { associated with acute kidney injury } \\
\text { after liver transplantation }\end{array}$ & $\begin{array}{l}\text { NADEEM, Ashraf et al., / } \\
2014\end{array}$ & $\begin{array}{l}\text { - Na amostra de pacientes submetidos ao TF, percebeu-se que } \\
\text { a maior infusão de fluidos com cloreto relacionou-se com uma } \\
\text { maior chance de IRA no pós-operatório. }\end{array}$ \\
\hline $\begin{array}{l}\text { Perioperative Calcium, Magnesium, } \\
\text { and Phosphorus Levels in Live } \\
\text { Donors For Liver Transplant }\end{array}$ & $\begin{array}{l}\text { MAHMOUD, Ahmed A. A. } \\
\text { et al., / } 2015\end{array}$ & $\begin{array}{l}\text { - Hipocalcemia, hipomagnesemia e hipofosfatemia são distúrbios } \\
\text { frequentes nos pacientes após TF, porém não mostrou uma } \\
\text { grande determinação para mortalidade deles. }\end{array}$ \\
\hline $\begin{array}{l}\text { Nutritional therapy in liver } \\
\text { transplantation. }\end{array}$ & $\begin{array}{l}\text { HAMMAD, Ahmed et al. / } \\
2017\end{array}$ & $\begin{array}{l}\text { - A maioria dos pacientes submetidos ao TF apresentam } \\
\text { destruição proteica, que aumenta a mortalidade e morbidade. }\end{array}$ \\
\hline $\begin{array}{l}\text { Postoperative Care of the Liver } \\
\text { Transplant Recipient }\end{array}$ & $\begin{array}{l}\text { PAREKH, Krishna N et al. / } \\
2017\end{array}$ & $\begin{array}{l}\text { - Alterações de sódio são as mais frequentes em pacientes } \\
\text { transplantados de fígado. Hipocalcemia costuma estar presente, } \\
\text { assim como hipocalemia. }\end{array}$ \\
\hline $\begin{array}{l}\text { Predictors of Infection and } \\
\text { Mortality in Living Donor Liver } \\
\text { Transplantation. }\end{array}$ & AMR, M. et al. / 2018 & $\begin{array}{l}\text { - A taxa de infecção em doador vivo foi de quase } 70 \% \text {, com } \\
\text { maioria no } 1 \text { o mês de pós-operatório. } \\
\text { - A taxa de infecção possui relação com longo tempo operatório. } \\
\text { A maioria das infecções aumenta a mortalidade. }\end{array}$ \\
\hline $\begin{array}{l}\text { Prevalência e fatores de risco } \\
\text { associados à hipercalemia após o } \\
\text { transplante hepático. }\end{array}$ & RIBEIRO, Helem et al., 2018 & $\begin{array}{l}\text { - Hipercalemia esteve presente em quase } 20 \% \text { dos pacientes no } \\
\text { pós-operatório do TF. }\end{array}$ \\
\hline $\begin{array}{l}\text { Abordagem ao período pós- } \\
\text { operatório inicial no transplante } \\
\text { de fígado: um ponto de vista } \\
\text { institucional. }\end{array}$ & $\begin{array}{l}\text { AMARAL, Beatriz et al. / } \\
2019\end{array}$ & $\begin{array}{l}\text { - A maior parte dos desequilíbrios após o TF ocorre na fase } \\
\text { de reperfusão, e as complicações mais comuns: disfunção } \\
\text { precoce do enxerto, rejeição, complicações da técnica cirúrgica, } \\
\text { complicações arteriais, biliares, hemodinâmicas, respiratórias e } \\
\text { infecções. }\end{array}$ \\
\hline $\begin{array}{l}\text { Potassium levels after liver } \\
\text { reperfusion in adult patients } \\
\text { undergoing cadaveric liver } \\
\text { transplantation: A retrospective } \\
\text { cohort study }\end{array}$ & $\begin{array}{l}\text { WEINBERG, Laurence et al., } \\
2020\end{array}$ & $\begin{array}{l}\text { - Hipercalemia está presente em } 1 \text { a cada } 2 \text { pacientes, } \\
\text { especialmente após a reperfusão no TF. Possivelmente os níveis } \\
\text { de potássio pré-operatório tem influência. }\end{array}$ \\
\hline
\end{tabular}

$* T F=$ Transplante de fígado; $* S I G=$ Strong Ion Gap; $p H=$ Potencial hidrogênico; *UTI = Unidade de terapia intensiva

Os distúrbios acidobásicos foram avaliados em $22,22 \%$ (4/18) dos artigos, demonstrando a superioridade do método de Stewart na avaliação dessas alterações em relação ao método tradicional, bem como a frequência da acidose metabólica nos pacientes em pós-operatório de TF. Foram descritas também as correlações entre a acidose e o aumento dos níveis de cloreto e de outros íons nesses pacientes. ${ }^{4,9,10} \mathrm{~A}$ hipoalbuminemia foi citada em $11,11 \%$ (2/18) dos artigos, apresentando relação com o estado de acidose e com a hipercloremia presente nos pacientes após o TF.4,11

Dos artigos, 16,66\% (3/18) levantaram a hipótese de que a infusão de soluções salinas com cloreto e ácidos fracos durante o perioperatório dos transplantes poderia contribuir para o desenvolvimento da acidose metabólica e hipercloremia nos pacientes. ${ }^{10-12}$ Além disso, um desses artigos trouxe também uma associação entre a quantidade de infusão de soluções com cloreto no perioperatório e o desenvolvimento de insuficiência renal aguda (IRA) nos pacientes em pós-operatório imediato. ${ }^{12}$

O estado de hiperlactatemia esteve presente em $11,11 \%$ (2/18) dos artigos, sendo descrito como um distúrbio frequente nos pacientes em pós-operatório imediato de TF. O aumento nos níveis de lactato foi relacionado com possíveis perdas sanguíneas no perioperatório, e com complicações como IRA e aumento da mortalidade nesses pacientes. ${ }^{13,14}$ 
As principais alterações hidroeletrolíticas foram descritas em 22,22\% (4/18) dos artigos e, dentre elas, as mais frequentes e preocupantes pelas complicações que podem causar são a hiponatremia e hipercalemia, estando essa última presente, em média, em $50 \%$ dos pacientes. Além dessas, a hipocalcemia, hipomagnesemia e hipofosfatemia são distúrbios de desenvolvimento comum no pós-operatório imediato de TF, mas menos preocupantes quanto ao impacto na mortalidade, em comparação aos primeiros ${ }^{3,15-17}$

\section{DISCUSSÃO}

O transplante de fígado é a alternativa de tratamento utilizada em muitos pacientes com doença hepática aguda ou crônica irreversível. Diante de seus avanços, com maior sobrevida e menor rejeição, seus níveis de sucesso têm aumentado, mas ainda existem desafios.
As infecções sempre foram a maior dificuldade no pós-operatório, mas alterações hidroeletrolíticas e acidobásicas no pós-operatório imediato do transplante também são intercorrências que merecem excessiva atenção. $^{5}$

A forma de avaliar a presença dos distúrbios acidobásicos em pacientes mudou ao longo do tempo e com o aprimoramento de técnicas e estudos. Anteriormente, usava-se o modelo tradicional de avaliação dos distúrbios acidobásicos que classifica a acidose metabólica com base no Standard Base Excess (SBE) como menor ou igual a $-5 \mathrm{mmol} / \mathrm{L}$. Já o modelo Stewart avalia esses distúrbios baseado em outro parâmetro, o Strong Ion Difference effective (SIDe), no qual a acidose metabólica é caracterizada por um SIDe $<38 \mathrm{mmol} / \mathrm{L}$ ou em caso de aumento de ácidos-fracos não voláteis.9 $\mathrm{A}$ comparação dos dois modelos de avalição encontra-se na tabela 2 , abaixo.

Tabela 2: Comparação dos modelos de avaliação dos distúrbios ácido-básicos

\begin{tabular}{|c|c|}
\hline Modelo tradicional & Modelo físico-químico \\
\hline Parâmetros: & Parâmetros: \\
\hline $\mathrm{PCO}_{2}, \mathrm{HCO}_{3}, \mathrm{SBE}^{*}$ e AG*. & $\mathrm{SID}^{*}, \mathrm{~A}_{\text {tot }}^{*}$ \\
\hline
\end{tabular}

* Standard Base Excess (SBE);

* Anion Gap $(A G)$ = a diferença entre cátions (sódio) e ânions (bicarbonato de sódio);

* SID = Strong ion difference $=$ a diferença de ions fortes, diferença de ânions e cátions plenamente dissociados

$\left(\left[\mathrm{Na}^{+}\right]+\left[\mathrm{K}^{+}\right]+\left[\mathrm{Ca}^{+}\right]+\left[\mathrm{Mg}^{+2}\right]-\left[\mathrm{Cl}^{-}\right]-\right.$[outros ânions fortes];

$* A_{\text {tot }}=$ a concentração total de ácidos fracos não-voláteis, especialmente albumina e fosfato, e a PCO2

De modo inicial, a avaliação dos distúrbios acidobásicos através do método tradicional leva em consideração os valores de: PCO2, HCO3, SBE e Anion Gap (AG), a diferença entre cátions (sódio) e ânions (bicarbonato de sódio) no sangue. Apesar de ser o método mais utilizado por sua simplicidade, acaba podendo modificar de complexas a simplórias as alterações em pacientes graves. Em contrapartida, o método de Stewart aplica três variáveis para avaliação do $\mathrm{pH}$, e consequentemente, dos distúrbios acidobásicos: strong ion difference (SID), a diferença de íons fortes, diferença de ânions e cátions plenamente dissociados $\left(\left[\mathrm{Na}^{2+}\right]+\left[\mathrm{K}^{+}\right]+\left[\mathrm{Ca}^{2+}\right]\right.$ $+\left[\mathrm{Mg}^{+2}\right]-[\mathrm{Cl}-]-$ [outros ânions fortes], a concentração total de ácidos fracos não-voláteis (Atot), especialmente albumina e fosfato, e PCO2 A partir de uma análise mais profunda, a quantificação, avaliação e descrição dos distúrbios são melhores através desse modelo. ${ }^{9}$
Nas análises dos distúrbios acidobásicos pósoperatórios imediatos do transplante hepático, o modelo físico-químico de Stewart tem sido majoritariamente utilizado em substituição ao método tradicional. Em um desses estudos realizado de modo prospectivo com 52 pacientes, foram analisados: diferenças de íons inorgânicos $\left(\mathrm{Na}^{+}+\mathrm{K}^{+}+\mathrm{Mg}^{2+}+\right.$ $\mathrm{Ca}^{2+}-\mathrm{Cl}^{-}$), lactato, ânions não mensurados (ânions presentes no plasma mesmo após o cálculo do SID e da concentração de ácidos fracos, demonstrando que o plasma não é eletricamente neutro), fosfato e albumina.

Os resultados mostraram prevalência de acidose metabólica em mais de $70 \%$ dos pacientes no pósoperatório imediato, e sua forma severa foi apresentada em quase $50 \%$ deles, enquanto apenas um paciente apresentou alcalose metabólica. ${ }^{4}$ 
A acidose metabólica, quando avaliada dentro desse modelo, apresenta diversas variáveis que a influenciam, e devido a isso, seu desenvolvimento é bastante complexo. Em linhas gerais, ela pode ser classificada em: acidose de baixo SID, com quantidades de cátions totalmente dissociados no plasma maior que os ânions, e acidose de alto strong ion gap (SIG), que significa a diferença entre o SID e as contribuições dos ácidos fracos no plasma, e que ao ser positivo, indica quantidade elevada de ânions não mensurados no plasma. Diferente do método tradicional, que valoriza muito o AG, o SIG leva em conta em seu cálculo, os valores de albumina, fosfato e lactato (ácidos fracos não-voláteis). Uma acidose mista com baixo SID e alto SIG, segundo estudo, possivelmente possui relação com hiponatremia, hipercloremia e retenção de ânions não mensurados (ácido lático, sulfato, acetato e gluconato). Nesse mesmo estudo foi associada acidose hiperclorêmica e acidose de outro íon ao uso de soluções com cloreto e ácidos fracos, sendo a segunda apenas detectável através do método de Stewart. ${ }^{10}$

Em outro estudo, demonstrou-se que pacientes em pós-operatório imediato de transplante de fígado desenvolveram também majoritariamente, acidose metabólica, que pode ser explicada por uma queda no SID com elevação de cloro, desenvolvendo dessa forma uma acidose hiperclorêmica. O efeito de uma hiponatremia também explica a queda do sódio no plasma, contribuindo para o distúrbio. Outra explicação para esse distúrbio é a presença de ânions não mensurados, expressos por um SIG positivo. Explicado novamente por um provável uso de soluções com cloro e ácidos fracos, desenvolvendo-se uma acidose metabólica hiperclorêmica e uma acidose de outro íon. Apesar de todo o padrão acidificante, a hipoalbuminemia, típica de pacientes candidatos ao TF, promove efeito contrário, um efeito alcalinizante, atenuando o distúrbio. ${ }^{11}$

Portanto, a acidose metabólica pode ser explicada por fatores como queda na diferença de íons inorgânicos (diferença de íons fortes, retirando lactato), aumento de ânions não mensuradas (sulfato, cetoácidos, citrato, piruvato, acetato e gluconato) e hipercloremia (aumento de $\mathrm{Cl}$ - que provoca diminuição do SID, causando acidose) presente nesses pacientes. Hipóteses quanto à relação entre aumento do cloro e da diferença dos íons inorgânicos pelo uso de soluções salinas no perioperatório foram levantadas. Em direção contrária, a hipoalbuminemia que ocorre, impacta de forma contrária na acidose, em estímulo à alcalose. ${ }^{4}$

O estado pós-operatório imediato de pacientes transplantados é de inflamação, aumento de permeabilidade dos capilares e mudanças na distribuição dos fluidos.
Devido a isso, é importante a monitorização dos níveis dos fluidos, de forma a evitar hipotensão. Por causa disso, a hipotensão é a complicação inicial mais recorrente nesses pacientes, devido à elevação do débito cardíaco, baixa resistência vascular periférica, hipovolemia e perda sanguínea, todas características do estado inflamatório. ${ }^{6}$

O lactato é um metabólito importante de sinalização quanto a hipoperfusão e hipóxia, além de ser um preditor de falência múltipla de órgãos e piores prognósticos. No pós-operatório da cirurgia de TF, a maioria dos pacientes apresenta baixa depuração desse metabólito, devido às complicações relacionadas à função hepática, marcada por hipóxia, hipoperfusão tecidual e acidose. ${ }^{13} \mathrm{Um}$ estudo que avaliou a concentração de lactato arterial no pós-operatório imediato em 48 pacientes submetidos ao TF demonstrou níveis de lactato acima dos valores de referência em quase $70 \%$ dos pacientes. Quanto mais altos os níveis desse metabólito, as complicações como injúria renal aguda e disfunção de enxerto aumentavam expressivamente. Além disso, a mortalidade dos pacientes foi diretamente proporcional aos níveis de lactato. $^{14}$

As complicações renais chamam atenção pelas várias etiologias, sendo a maior agente causadora dessas complicações a má distribuição dos fluidos. Uma disfunção renal grave promove retenção de fluidos, que gera distúrbios como acidose e alterações hidroeletrolíticas. 6 Percebeu-se em alguns estudos uma associação entre a frequência de hipercloremia nos pacientes em pósoperatório de TF e o desenvolvimento de insuficiência renal aguda (IRA). Na realidade, analisou-se que a grande quantidade de infusão intravenosa de soluções de cloreto no intra-operatório gerava hipercloremia nos pacientes, que posteriormente desenvolviam IRA. Além da hipercloremia, esses pacientes também apresentam alta frequência de acidose metabólica. ${ }^{12}$

O estado pós-operatório imediato do transplante de fígado é multifatorial, dependente do estado préoperatório do paciente, das administrações de fluidos e de sangue durante a cirurgia, como alguns estudos já sugeriram. ${ }^{7}$ Pacientes cirróticos, por exemplo, submetidos ao transplante e em largo uso de diuréticos, podem desenvolver hipofosfatemia, hipomagnesemia e hipocalemia, como reflexo de seu estado préoperatório. ${ }^{18}$

O sódio é um dos íons mais alterado no pós-operatório, possivelmente pela relação com a osmolaridade plasmática. É provável que as administrações de soluções cristaloides durante a cirurgia de transplante impactem nos níveis plasmáticos de sódio, apesar de 
sua alteração ser multifatorial. ${ }^{8}$ Dentre os distúrbios, a hipernatremia é a menos frequente, muitas vezes associada ao uso de laxantes osmóticos, como a lactulose. Já a hiponatremia é excessivamente preocupante pela possibilidade do desenvolvimento de complicações neurológicas. ${ }^{3}$

O potássio é um eletrólito importante antes, durante e após a cirurgia de TF. ${ }^{15}$ Ele pode ocasionar distúrbios de polarização e despolarização, como arritmias, além de alterações na contratilidade miocárdica. ${ }^{16} \mathrm{O}$ aumento nos níveis de potássio é a complicação mais preocupante nos pacientes transplantados de fígado, devido à sua maior mortalidade. ${ }^{3}$ Esse distúrbio, a hipercalemia, especialmente após a reperfusão do fígado transplantado, está presente em um a cada dois pacientes. ${ }^{15}$ No pós-operatório, ele está presente, em média, em $20 \%$ dos pacientes, possuindo relação com o uso de imunossupressores, hipertensão, distúrbios renais e acidose..$^{16}$
Além dos eletrólitos já exemplificados, o cálcio e o magnésio também demonstram alterações importantes no pós-operatório imediato do TF. Em um estudo retrospectivo com 44 pacientes, a maioria deles apresentou valores séricos de cálcio e magnésio na faixa abaixo da referência, apresentando hipocalcemia e hipomagnesemia. O fósforo, também analisado, apresentava-se no limite inferior de normalidade. ${ }^{17}$ Os níveis mais baixos de cálcio são usualmente encontrados nesses pacientes em pós-operatório imediato de TF. ${ }^{3}$

\section{CONCLUSÃO}

A acidose metabólica, segundo o modelo físico-químico de Stewart, associada à hipercloremia, hiponatremia, hipocalcemia e hipercalemia são os distúrbios mais comuns no pós-operatório imediato do TF. O maior conhecimento na utilização de fluidos no perioperatório promoverá maior equilíbrio do meio interno, levando a resultados melhores nos receptores de TF.

\section{RESUMO}

Introduction: The immediate postoperative period of liver transplantation (LT) is an instable moment that needs control and patient monitoring. Hidroelectrolytic and acid-basic alterations are just one among many complications in the immediate postoperative of LT, but draws the attention, and requiring a quick and correct handling. Purpose: The aim of this review is to assess the main acid-basic and hidroeletrolytic alterations in the immediate postoperative period of patients after LT. Method: This is a review, a qualitative study; the research was performed on the MEDLINE databases, through the National Center of Biotechnology Information, by acessing the Pubmed website. The inclusion criteria were: (1) publications in English, (2) to be an original article or literature review, and (3) to be in agreement with the theme. Results: According to the articles analyzed, around above $70 \%$ patients presented metabolic acidosis on the immediate postoperative of LT, associated to hypercloremia. Sodium levels are the most altered, with hyponatremia. On average $20 \%$ of patients presented hyperkalemia after LT. Hypocalcemia, hypomagnesemia and hypophosphatemia are frequent in these patients. Conclusion: Metabolic acidosis associated to hypercloremia, hyponatremia, hypocalcemia and hyperkalemia are the most common disorders on the immediate postoperative period of LT.

Keywords: Acid Base Imbalance; Water Electrolyte Imbalance; Postoperative Period; Liver Transplantation;

\section{AGRADECIMENTOS}

Agradecemos a todos que contribuíram para que fosse possível desenvolver essa revisão, e assim concretizá-la. 


\section{REFERÊNCIAS}

1. Ceneviva R, Vicente YAMVAV. Equilíbrio hidroeletrolítico e hidratação no paciente cirúrgico. Medicina (B Aires). 2008;41(3):283-296.

2. Moriya T, Martins ACP, Cherri J, Piccinato CE, Okano N, Carneiro JJ, et al. Hidratação e equilíbrio ácido-base em pacientes cirúrgicos. Acta Cir Bras [Internet]. 2000 [cited $2021 \mathrm{Jul}$ 8];15(suppl 2):34-8. Available from: http:// www.scielo.br/scielo.php?script=sci_arttext\&pid=S0102$86502000000600011 \&$ Ing $=$ pt\&tlng $=$ pt

3. Parekh KN, Crowley JC, Liu LL. Anesthesia and Perioperative Care for Organ Transplantation. Anesth Perioper Care Organ Transplant. 2017;

4. Boniatti MM, Filho EMR, Cardoso PRC, Vieira SRR. Physicochemical evaluation of acid-base disorders after liver transplantation and the contribution from administered fluids. Transplant Proc [Internet]. 2013;45(6):22837. Available from: http://dx.doi.org/10.1016/j. transproceed.2013.03.044

5. AMR M. AZIZ, M.D. WSMMD., EL-SHARNOBY, M.D. A. Predictors of Infection and Mortality in Living Donor Liver Transplantation. Med J Cairo Univ. 2018;86(6):2035-43.

6. Amaral B, Vicente M, Pereira CSM, Araújo T, Ribeiro A, Pereira R, et al. Approach to the liver transplant early postoperative period: An institutional standpoint. Rev Bras Ter Intensiva. 2019;31(4):561-70.

7. Feltracco P, Barbieri S, Galligioni H, Michieletto E, Carollo C, Ori C. Intensive care management of liver transplanted patients. World J Hepatol. 2011;3(3):61-71.

8. Faenza S, Bernardi E, Cuppini F, Gatta A, Lauro A, Mancini E, et al. Intensive care complications in liver and multivisceral transplantation. Transplant Proc. 2005;37(6):2618-21.

9. Boniatti MM. Avaliação diagnóstica e prognóstica dos distúrbios ácido-básicos em pacientes críticos pelo método de Stewart. Programa Pós-Graduação em Med - Ciências Médicas Univ do Rio Gd do Sul, Porto Alegre [Internet]. 2010;(Tese (Doutorado em Medicina) - Universidade Federal do Rio Grande do Sul, Porto Alegre). Available from: http://hdl.handle.net/10183/27813
10. Ali $\mathrm{Y}$, Abouelnaga S, Khalaf H, Kamel Y. Physical chemical approach versus traditional technique in analyzing blood gases and electrolytes during liver transplant surgery. Transplant Proc [Internet]. 2010;42(3):861-4. Available from: http://dx.doi. org/10.1016/j.transproceed.2010.03.003

11. Story DA, Vaja R, Poustie SJ, McNicol L. Fencl-Stewart analysis of acid-base changes immediately after liver transplantation. Crit Care Resusc. 2008;10(1):19-23.

12. Nadeem A, Salahuddin N, El Hazmi A, Joseph M, Bohlega B, Sallam $\mathrm{H}$, et al. Chloride-liberal fluids are associated with acute kidney injury after liver transplantation. Crit Care. 2014;18(6):1-8.

13. Basile-Filho A, Nicolini EA, Auxiliadora-Martins M, Silva Jr O de $\mathrm{C}$ e. The use of perioperative serial blood lactate levels, the APACHE II and the postoperative MELD as predictors of early mortality after liver transplantation. Acta Cir Bras. 2011;26(6):535-40.

14. Jipa LN, Tomescu D, Droc G. The interrelation between arterial lactate levels and postoperative outcome following liver transplantation. Rom J Anaesth intensive care. 2014;21(2):106-12.

15. Weinberg L, Lee DK, Koshy AN, Leong KW, Tosif $\mathrm{S}$, Shaylor R, et al. Potassium levels after liver reperfusion in adult patients undergoing cadaveric liver transplantation: A retrospective cohort study. Ann Med Surg [Internet]. 2020;55(May):111-8. Available from: https://doi.org/10.1016/j.amsu.2020.05.002

16. Ribeiro HS, Oliveira MC, Anastácio LR, Generoso SV, Lima AS, Correia MI. Prevalência e fatores de risco associados à hipercalemia após o transplante hepático. Arq Bras Cir Dig. 2018;31(1):1-4

17. Mahmoud AAA, El-Shaarawy AM, Mansour MA, Abdelhaq MM, Maher MA, Kamal AM. Perioperative calcium, magnesium, and phosphorus levels in live donors for liver transplant. Exp Clin Transplant. 2015;13(6):550-5

18. Hammad A, Kaido T, Aliyev V, Mandato C, Uemoto S. Nutritional therap 